\title{
Analysis of Sign Language Gestures using Size Functions and Principal Component Analysis
}

\author{
Daniel Kelly, John McDonald, Tom Lysaght, Charles Markham \\ N.U.I. Maynooth \\ Computer Science Dept. \\ Co. Kildare \\ dankelly@cs.nuim.ie
}

\begin{abstract}
This paper presents a computer vision based virtual learning environment for teaching communicative hand gestures used in Sign Language. A virtual learning environment was developed to demonstrate signs to the user. The system then gives real time feedback to the user on their performance of the demonstrated sign. Gesture features are extracted from a standard web-cam video stream and shape and trajectory matching techniques are applied to these features to determine the feedback given to the user.
\end{abstract}

\section{Introduction}

In today's world, communication is predominantly expressed in the form of voice sounds, but for anyone with an inability to communicate using voice an alternative form of communication such as sign language is needed. It is a general rule of thumb that 1 in every 1000 people are deaf. The majority of deaf people rely on Sign Language as their alternative form of communication; however, because the number of people, in the general community, who understand sign language is small, communication with people outside of the immediate deaf community can be extremely difficult.

The practical application of the work detailed in this paper is to provide the general community with a virtual environment for learning sign language. The virtual teaching environment provides an immersive learning experience while also retaining the flexibility of learning at the users convenience on a standard PC. The system was developed with consultation and help from the Irish Deaf Society (www.irishdeafsociety.ie).

\section{Modeling Sign Language}

Sign language is often regarded as the most structured of the various gesture categories [1]. In sign language, the structure of the signs can be described as the combination of the configuration, position and movement of the hand [2]. This work follows the basic ideas of a segmental model known as the Movement-Hold model [3]. The movementhold model breaks a sign into a sequence of segments, were a segment is either a movement or a hold. Movements are defined as those segments in which some aspect of the hand configuration changes, such as a change in hand shape, hand movement or hand orientation. Holds are defined as those segments during which all aspects of the signs configuration remain unchanged for a brief period of time. Signs are made up of sequences of movements and holds.

The system described here extracts features describing hand shape, position and movement from a standard webcam video stream and then applies Sizemap [4][5] and Principal Component Analysis (PCA) techniques to these features to give real-time feedback on sign performance.

\section{Tracking and Feature Extraction}

Tracking of the hands is performed by tracking coloured gloves (see Fig. 1-a) using the Mean Shift algorithm [6]. To initialize tracking the start position of the two hands are specified. A sample region of $n \times n$ pixels is taken at these specified locations. For each of the two sample regions taken, a color histogram of the Hue component of the region is calculated. Using the camShift function (a variation on Mean Shift algorithm with an adaptive region-sizing step [7]) within the OpenCV API and the colour Histogram, a Colour Probability Distribution (see Fig. 1-b) is calculated and the angle and location of the object is determined. The tracking region is then scaled to fit the size of the object and shifted to the centroid of the Colour Probability Distribution 
for the next frame.

The key feature used for the analysis of the hand shape is the external contour made by the hand (see Fig. 1-c). To extract this feature from the image, the Colour Probability Distribution is used. A binary image with the same dimensions as the current tracking region is created and each pixel set to a $l$ if the corresponding pixel in the Colour Probability Distribution is above a set threshold. The external contour of this binary image is then calculated using cvFindContours.

A second feature used, to improve classifier accuracy, is the Hu set of image moments [8], which are invariant under translation, scale and rotation.

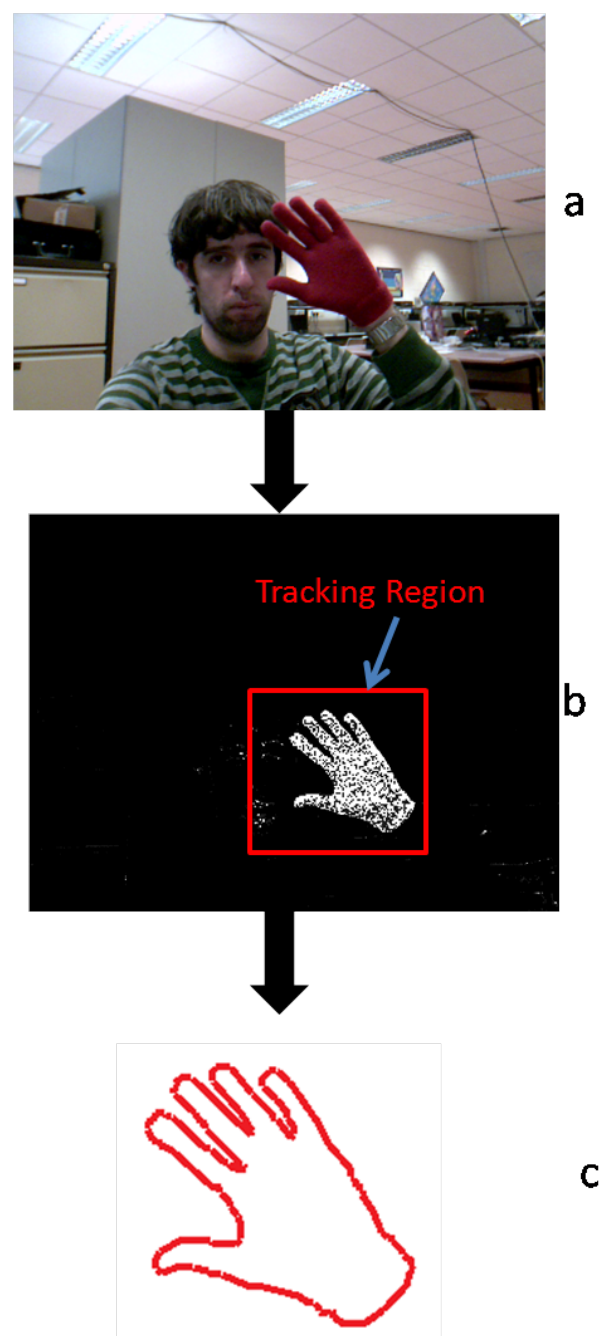

Figure 1. RGB Image to Hand External Contour

\section{Hand Shape Analysis}

As stated in [2] and following the movement-hold model, the structure of the signs can be described as the combination of the shape, position and movement of the hand. In order to give feedback on sign performance, analysis on these three sign components must be performed.

Hand shape analysis is performed by applying Size Function [4][5] pattern matching techniques on the external hand contour described in Section 3.

\subsection{Size Functions}

Size functions are integer valued functions which represent both qualitative and quantitative properties of a visual shape. To illustrate the concept of size functions and their application in analyzing hand shapes used in sign language, a specific example will be used. The shape of the hand shown in 2-A (the letter $\mathrm{k}$ in sign language) is described by the contour in Figure 2 which was obtained by the feature extraction method shown in Section 3. For the given contour, let $G$ be the graph whose vertices are the points of the contour and whose edges connect the vertices which correspond to adjacent points on the contour. Let $\varphi$ be any real valued function, called the measuring function, defined on the vertices of $\mathrm{G}$. The application described in this work uses a measuring function $\varphi=D_{0}(p)$ : a function which measures the distance between the horizontal axis and a point $p$, which is above the horizontal axis, on the graph $G$. The size function $\ell_{\varphi}$ induced by the measuring function $\varphi$, is an integer valued function defined on a real pair $(x, y)$ according to the following algorithm.

1. Find subgraph $G_{\varphi \leq y}$ of $G$;

2. Identify the connected components of $G_{\varphi \leq y}$ (see Figure 2-D);

3. The size function $\ell_{\varphi}$ at the point $(x, y)$ equals the number of connected components of $G_{\varphi \leq y}$ which contain at least a vertex with $\varphi(p) \leq x$ (see Figure 2-E);

So far it has been shown that the contour above the horizontal axis is only taken into consideration when applying the measuring function $D_{0}$. The size function model implemented in this system uses a family of measuring functions indexed by the angle $\theta\left(0 \leq \theta \leq 360^{\circ}\right)$, were the measuring function $D_{0}$ is a particular case of $D_{\theta}$ were $\theta=0$. The size functions for $D_{\theta}$ are computed by two steps; first the contour is rotated about its centre of gravity by an angle $\theta$ and then the function $D_{0}$ is calculated on the rotated contour.

A comparison of two hand shapes can now be achieved by comparing each size function generated from one hand $\ell_{D_{\theta}}^{1}$ with the corresponding size function generated from the 


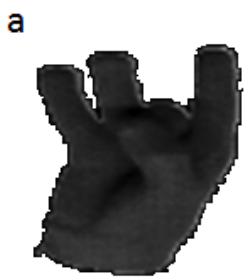

b

C

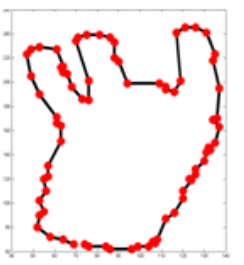

e

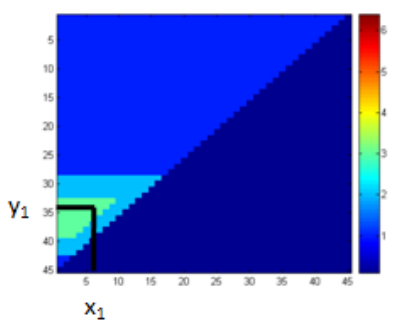

Figure 2. Size Functions. (A). A segmented image of the $k$ sign. (B). Contour of $k$ sign. (C). Graph associated with subsampled points of (B). (D). Measuring function applied to (C) with $D \leq y_{1}$. (E). Diagram of the size function $\ell_{D_{0}}(x, y)$.

second hand $\ell_{D_{\theta}}^{2}$. Comparison can be achieved by, for example, calculating the euclidian distance between each $\ell_{D_{\theta}}^{1}$ and $\ell_{D_{\theta}}^{2}$ for every $\theta$. For the application being focused on in this work (i.e. giving feedback on users performance of a particular sign), it is necessary to be able tell whether or not a users' hand is in the desired shape. Thus, the output of a comparison between two hand shapes must be a true or false result. In tests, the raw size functions and the euclidian distance between them were used as a metric. If the distance was below a set threshold then the hand shape was considered as matching the desired shape.

Tests showed, that even though the Euclidian distance was strong at determining true positives, the metric produced a high amount of false positives when presented with particular hand shapes that were not similar to that of the control hand shape. In an experiment, six hand shape contours were recorded, each representing a letter in the Irish Sign language alphabet (a,d,f,k,i and l). For each hand shape, the Euclidian metric was used to compare the recorded contour with that of 20 test shapes (10 correct and 10 incorrect), were the incorrect test shapes were selected from signs that were seen to be similar to the shape it was being compared to under a subjective assessment. Results show that all of the correct hand shapes were classified as true, but $28.4 \%$ of the incorrect hand shapes were misclassified as true. It is to be noted that the high correct classification rate is a result of the variable threshold being set so that feedback is given when correct sign is recalled, not to allow the user to perfect the sign.

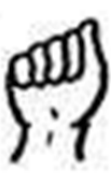

A

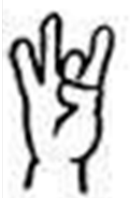

K

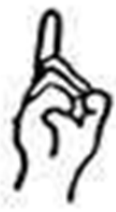

D

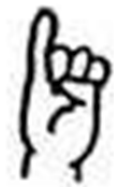

I

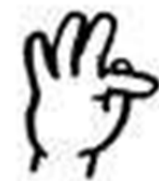

$\mathbf{F}$

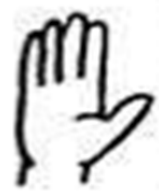

$\mathbf{L}$

Figure 3. ISL Alphabet letters a, d, f, k, i and I 


\subsection{PCA and Size Functions}

In order to make hand shape classification, using size functions, more robust an alternative method of comparison is proposed. This technique is a method of comparing the principal components of the size functions as calculated using Principal Component Analysis (PCA). PCA is a statistical technique used in face recognition, image compression and used for finding patterns in data of high dimensions. Since we are looking for similarities and differences between two size functions, PCA can be used to highlight these similarities and differences.

To calculate the principal components of a size function, the size function is described as an $N \times N$ matrix $\mathrm{X}$. The vector $\mathrm{u}$ is the empirical mean of $\mathrm{X}$ (see Equation 1), $\mathrm{B}$ is the mean subtracted $N \times N$ matrix (see Equation 2) and $\mathrm{C}$ is the covariance matrix of $\mathrm{B}$ (see Equation 3).

$$
\begin{gathered}
u[m]=\frac{1}{N} \sum_{n=1}^{N} X[m, n] \\
B=X-[u, u, \ldots . ., u] \\
C=\frac{1}{N} B \cdot B^{T}
\end{gathered}
$$

The eigenvectors and eigenvalues of $C$ are calculated according to Equation 4 were $v$ is the eigenvector and $w$ is the eigenvalue associated to the eigenvector.

$$
C v=w v
$$

The columns of the eigenvector matrix $V$ and the eigenvalue matrix $W$ are sorted in order of decreasing eigenvalue. This gives the components in order of significance, the eigenvector with the highest eigenvalue being the principal component. To perform a comparison of the size function, we generate the eigenvectors and eigenvalues for that size function, and choose only the first $\mathrm{P}$ eigenvectors, resulting in a matrix $\mathrm{M}$ with dimensions $N \times P$. A euclidian distance is then calculated between each eigenvector for the test hand shape $\left(H_{1}\right)$ and its corresponding eigenvector for the control hand shape $\left(H_{2}\right)$.

$$
\delta_{12}=\sum_{\theta=0}^{360} \sum_{p=0}^{P} \sum_{i=0}^{N} M_{\theta 1}[p, i]-M_{\theta 2}[p, i]
$$

Were $\theta$ is a fixed set of $c$ evenly distributed angles $\theta$ were $\left(0 \leq \theta \leq 360^{\circ}\right)$ (e.g. for $\left.c=4, \theta=\left[0^{\circ}, 90^{\circ}, 180^{\circ}, 270^{\circ}\right]\right)$, and $M_{1}$ and $M_{2}$ are the eigenvectors for the hand shape $H_{1}$ and $\mathrm{H}_{2}$ respectively.

To measure the accuracy of the PCA classifier described above, the same experiment, that was applied to the raw size function euclidian metric, was applied to the PCA metric.
That is, six hand shapes were recorded (a,d,f,k,i and l) and the PCA classifier was used to classify 20 test hand shapes (10 correct and 10 incorrect). As with the raw size function euclidian metric, all of correct hand shapes were classified as true, but the PCA classifier only misclassified $11.6 \%$ of incorrect hand shapes as true, as opposed to $28.4 \%$ with the raw size function euclidian metric.
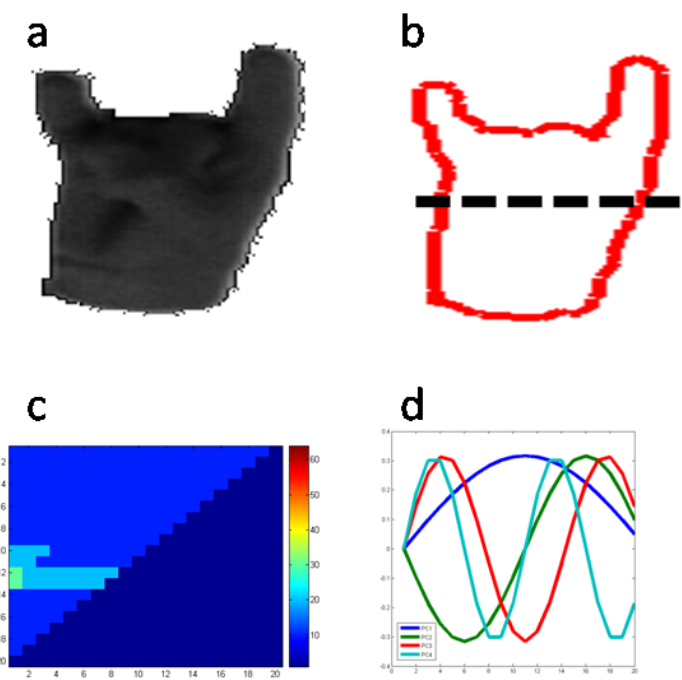

Figure 4. Eigenvectors of Size Function. (A). A segmented image of the $h$ sign. (B). Contour of $h$ sign. (C). Size function of above horizontal axis portion of (B). (D). Eigenvectors of $(B)$ with the four highest eigenvalues

\subsection{Hu Moments}

To increase classification accuracy the Hu set of seven invariant moments [8] are extracted from a binary image of a particular hand shape. A comparison of the Hu Moments, extracted from two hand shapes, will result in $v$ were $(0 \leq$ $v \leq 1)$. 0 will be the result of two exactly the same hand shapes.

$v_{i j}$ is used in conjunction with $\delta_{i j}$ to improve classification as in Equation 6, were $i$ and $j$ are indices of particular hand shapes and $\mathrm{w}$ is a weight applied to $v_{i j}$.

$$
\xi_{i j}=\delta_{i j} *\left(v_{i j} * w\right)
$$

\subsection{Hand Movement Analysis}

So far, only the analysis of the hand shape has been addressed, which corresponds to the features required to ana- 
lyze hands when in a hold segment of a sign. The second component of a gesture which must be analyzed is that of the movement segments. As described in Section 3 the position of the hands' centre of mass, angle and area is recorded at each frame. Over the entire sequence, each of these features are recorded in separates lists $\Gamma, \Phi$ and A respectively.

To analyze hand movement, subsets of $\Gamma$ must be found such that each subset maps to a movement segment of a sign. As described in Section 2 the movement segments are defined as those segments in which some aspect of the hand configuration changes, such as a change in hand shape, hand movement or hand orientation, or alternatively, the sign is in a movement segment if it is not in a hold segment. Holds are defined as those segments during which all aspects of the signs configuration remain unchanged for a brief period of time.

$$
\eta_{t}=\frac{d \Gamma(t)}{d t}+\frac{d \Phi(t)}{d t}+\frac{d A(t)}{d t}
$$

Equation 7 defines a value $\eta_{t}$ that represents the total movement of the hand at time t. If $\eta_{t}$ is below a set threshold then the hand is defined as being in a hold segment, otherwise, the hand is in a movement segment. The time when a hand enters a movement segment, $t_{i}$, and the time it stops being in that movement segment, $t_{i+1}$, is recorded so analysis of the movement within these time periods can be performed. The current movement segment is defined in Equation 8.

$$
\Gamma_{i}=\left[\Gamma\left(t_{i}\right), \Gamma\left(t_{i}+1\right), \Gamma\left(t_{i}+2\right), \ldots \ldots, \Gamma\left(t_{i+1}\right)\right]
$$

To compare two movement sequences, $\Gamma_{i}$ and $\Gamma_{j}, \Gamma_{i}$ must firstly be interpolated so that the length of $\Gamma_{i}$ equals the length of $\Gamma_{j}$. Each sequence is then translated such that the centre of mass of each sequence lies on the origin. Then each of the sequences, $\Gamma_{i}$ and $\Gamma_{j}$, is converted into polar co-ordinates $\Theta_{i}, D_{i}$ and $\Theta_{j}, D_{j}$, were $\Theta$ is the sequence of polar angles and $\mathrm{D}$ is the sequences of polar distances.

$$
\begin{gathered}
\Delta D_{i j}=\frac{1}{T} \sum_{t=0}^{T}\left(\frac{d D_{i}(t)}{d t}-\frac{d D_{j}(t)}{d t}\right)^{2} \\
\Delta \Theta_{i j}=\frac{1}{T} \sum_{t=0}^{T}\left(\Theta_{i}(t)-\Theta_{j}(t)\right)^{2}
\end{gathered}
$$

Using Equations 9 and 10 the differences between the two movement sequences can be calculated. The sequences are determined to be of similar movement if both $\Delta D_{i j}$ and $\Delta \Theta_{i j}$ are below a set threshold.

\section{Implementation and Performance}

The system described in this work was developed as a robust software application that can be used on any standard
Microsoft Windows based PC. The vision feature extraction component of the system was built using $\mathrm{C}++$ and utilizes OpenCV functions. The user interface (see Figure 5) and feature analysis engine was developed using C\# and using a Direct 3D avatar to demonstrate signs to the user. The system was built using object oriented programming and is a working application that has been demonstrated live in lecture halls. The software has been awarded fourth place in a worldwide software development competition (Microsoft Imagine Cup).

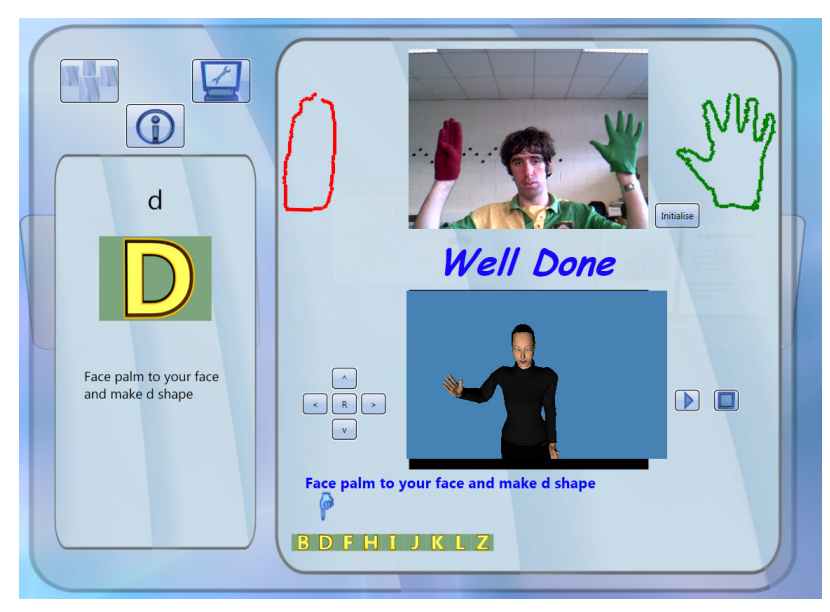

\section{Figure 5. Front End of Sign Language Teach- ing Software with 3D avatar}

Tests were performed to calculate the total speed of the system. Results show that the average time, of feature extractions, feature analysis and feature comparison was 60 milliseconds, which results in the average frames per second being $16.7 \mathrm{fps}$.

To test the accuracy of the system, an experiment was carried out in which two subjects were asked to perform the signs b, d, f, h, i, j, k, 1 and $\mathrm{z}$ from the Irish Sign Language alphabet. Each subject was asked to perform each of the 9 signs 7 times. It is to be noted that the signs for the letter $\mathrm{j}$ and the letter $\mathrm{z}$ are signs which contain both hold and movement segments (See Figure 6). Results of the experiment show the system classifies $93.8 \%$ of correct signs as correct while only classifying $9.1 \%$ of incorrect signs as correct. The aim of the system is to give feedback on sign performance and learning, and from the results of the above experiment it can be seen the system performs at a promising level in giving correct feedback. 


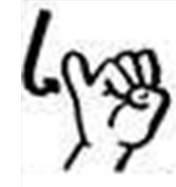

J

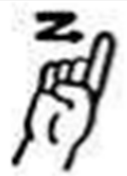

$\mathbf{Z}$
Figure 6. $\mathrm{J}$ and $\mathrm{Z}$ signs from ISL alphabet

\section{Conclusions}

This paper has described how a system has been developed to teach sign language by giving real time feedback on sign performance. It was shown how sign features were extracted from a video stream and how these features, used in conjunction with the Movement-Hold model of signing, were analyzed and how a users' sign performance is compared to that of a fluent signers'.

It was shown how size functions can be applied to hand shape contours and a method of comparing size function using PCA was proposed. A method of hand trajectory analysis and comparison was also proposed.

It was shown that the work described in the paper is realized in a working application which operates at $16.7 \mathrm{fps}$ and, in tests, has been shown to classify correct signs with 93.8\% accuracy.

This work described in this paper will be used to contribute to the overall research goal of recognizing continuous sign language

\section{Future Work}

The work described in this paper is being extended to fully classify a hand shape from a number of pre-recorded hand shapes. Early work on this is indicating a recognition accuracy of around $90 \%$ for the recognition of static alphabet sign from the Irish Language Alphabet.

This research will be continued with the goal of developing a full continuous sign language recognition system. Hidden Markov Models have been used frequently in attempts automate the recognition of continuous sign language [9][1], while Simple Recurrent Networks have been used to train language based systems on the grammar of a language [10]. Both these techniques are being researched with the view of extending the segmental model, used in this work, to a continuous model of sign language recognition.

\section{Acknowledgements}

The author would like to acknowledge the financial support of the Irish Research Council for Science, Engineering and Technology.

\section{References}

[1] Sylvie C. W. Ong and Surendra Ranganath. Automatic sign language analysis: A survey and the future beyond lexical meaning. IEEE Trans. Pattern Anal. Mach. Intell., 27(6):873-891, 2005.

[2] Jr. Stokoe, William C. Sign language structure: An outline of the visual communication systems of the american deaf. Journal of Deaf Studies and Deaf Education, v10 n1 p3-37 Win 2005, 2005.

[3] Johnson R.E. Liddell, S.K. American sign language: The phonological base. Sign Langauge Studies, 64.

[4] A. Uras, C.; Verri. On the recognition of the alphabet of the sign language through size functions. Pattern Recognition, 1994. Vol. 2 - Conference B: Computer Vision \& Image Processing., Proceedings of the 12th IAPR International. Conference on, 2:334-338 vol.2, 9-13 Oct 1994.

[5] A. Verri, C. Uras, P. Frosini, and M. Ferri. On the use of size functions for shape analysis. Qualitative Vision, 1993., Proceedings of IEEE Workshop on, pages 89-96, 14 Jun 1993.

[6] D. Comaniciu, V. Ramesh, and P. Meer. Real-time tracking of non-rigid objects using mean shift. Computer Vision and Pattern Recognition, 2000. Proceedings. IEEE Conference on, 2:142-149 vol.2, 2000.

[7] Gary R. Bradski. Computer vision face tracking for use in a perceptual user interface. Intel Technology Journal Q2'98.

[8] Ming-Kuei Hu. Visual pattern recognition by moment invariants. Information Theory, IEEE Transactions on, 8(2):179-187, Feb 1962.

[9] Ayush Parachar Barbare L. Loeding, Sudeep Sarkar and Arthur I. Karshmar. Progress in automated computer recognition of sign language. Lecture Notes in Computer Science, 3118.

[10] Jeffery L. Elman. Distributed representations, simple recurrent networks, and grammatical structure. Machine Learning, 7(2-3), 1991. 